\title{
Interaction of caldesmon with phospholipids
}

\author{
Edward A. CZURYK0, ${ }^{\star}$ Józef ZBOROWSKI, $†$ and Renata DABROWSKA $\ddagger$ \\ Departments of *Muscle Biochemistry and †Cellular Biochemistry, Nencki Institute of Experimental Biology, 3 Pasteur Str., 02-093 Warsaw, Poland
}

The interaction of caldesmon with liposomes composed of various phospholipids has been examined by tryptophan fluorescence spectroscopy. The results indicate that caldesmon makes its strongest complex with phosphatidylserine (PS) vesicles $\left(K_{\text {ass. }}=1.45 \times 10^{5} \mathrm{M}^{-1}\right)$. Both electrostatic and hydrophobic interactions contribute to the stability of this complex. The site for strong binding of PS seems to be located in the N-terminal part of the $34 \mathrm{kDa}$ C-terminal fragment of caldesmon. Binding of PS at this site results in displacement of calmodulin from its complex with caldesmon.

\section{INTRODUCTION}

It has been well-documented that many actin-binding proteins interact with lipids (for review see [1]). Recently the list of these proteins has been extended to include caldesmon [2].

Caldesmon, a protein originally found in smooth muscle [3], is also distributed in a wide variety of non-muscle cells, including many types of cultured cells [4], platelets [5], adrenal medulla cells [6] and T-lymphocytes [7]. It is believed that this protein is an important regulator of both motility and cell architecture $[8,9]$. The latter function comes from caldesmon-mediated control of actin assembly/disassembly.

It has been found previously that caldesmon interacts not only with filamentous actin but also with monomeric actin, which leads to its polymerization in the absence of salt $[10,11]$. The actin monomers can be recruited from profilactin, since caldesmon is capable of dissociating the actin-profilin complex [12]. The actin-binding ability of caldesmon is regulated by calmodulin, which in the presence of micromolar concentrations of $\mathrm{Ca}^{2+}$ forms a complex with caldesmon [9].

Immunofluorescent studies have shown that in cultured fibroblasts caldesmon is located along stress fibres and in the membrane ruffles [4]. Since the latter regions of the cell are also rich in profilin and actin [13] it is possible that all these proteins are involved in the formation of the dynamic structures of the subplasmalemmal cytoskeleton. This, in turn, raises the possibility that caldesmon, like profilin $[13,14]$, can interact with membranous lipids. The report of Vorotnikov and Gusev [2] showing interaction of caldesmon with soyabean azolectin is consistent with this idea.

In this work we have studied the interaction of smooth muscle caldesmon with various phospholipids in the absence and presence of calmodulin.

\section{MATERIALS AND METHODS}

\section{Preparation of proteins}

Caldesmon from chicken gizzards was prepared basically according to the procedure of Bretscher [15] with some modifications described previously [16]. Calmodulin was obtained from bovine brain by the method of Gopalakrishna and Anderson [17]. The homogeneity of these proteins was monitored by SDS/PAGE on $12.5 \%(\mathrm{w} / \mathrm{v})$ acrylamide slab gels according to the method of Laemmli [18].
Protein concentration was determined spectrophotometrically using absorption coefficient values of 0.38 for caldesmon [16] and 0.20 for calmodulin [17] at $278 \mathrm{~nm}$, each at a concentration of $1 \mathrm{mg} / \mathrm{ml}$ with a $1 \mathrm{~cm}$ light path. The molecular masses of caldesmon and calmodulin were taken to be $87 \mathrm{kDa}$ [19] and $16.7 \mathrm{kDa}[17]$ respectively.

\section{Purification of lipids}

Phosphatidylserine (PS) from bovine brain cortex was purified by extraction [20] followed by column chromatography on CMcellulose according to Comfurius and Zwaal [21]. Phosphatidylcholine (PC) from egg yolk was prepared as described by Singleton et al. [22]. Cardiolipin (CL) from bovine heart, and phosphatidic acid (PA) from egg yolk, were obtained from KochLight, Colnbrook, Bucks., U.K., while phosphatidylinositol (PI) from bovine liver was from Avanti Polar-Lipids, Alabaster, AL, U.S.A.

The purity of all phospholipids used was checked by t.l.c. using a two-dimensional solvent system described by Rouser et al. [23]. Each of the phospholipids co-migrated as a single spot with the respective standard. Phospholipid phosphorus levels were determined according to Rouser et al. [23].

\section{Preparation of liposomes}

Liposomes were prepared by sonication of dried lipid material in a buffer containing $20 \mathrm{mM}$ Tris/ $\mathrm{HCl}(\mathrm{pH} 7.5), 0.5 \mathrm{mM}$ EGTA, $1 \mathrm{mM} \mathrm{NaN}_{3}$, and $1 \mathrm{mM}$ 2-mercaptoethanol (buffer A), for $15 \mathrm{~min}$ under $\mathrm{N}_{2}$. The sonication tube was placed in an ice-bath and the length of each pulse from the Ultrasonic Disintegrator Model UD-11 (Techpan, Poland) working at maximum output was $30 \mathrm{~s}$, with a subsequent interval of equal length.

\section{Fluorescence measurements}

Steady-state tryptophan fluorescence measurements were made on a Perkin-Elmer spectrofluorimeter Model LS-5B equipped with a 3700 Data Station (Perkin-Elmer). All spectra were corrected for light scattering, solvent background and dilution. In all experiments an excitation wavelength of $297 \mathrm{~nm}$ and $5 \mathrm{~nm}$ slits were used. The intensities of the corrected spectra were proportional to the number of photons emitted per unit wavelength interval. Relative quantum yield $(Q)$ was calculated as the ratio of the quantum yield of tryptophan fluorescence of caldes- 

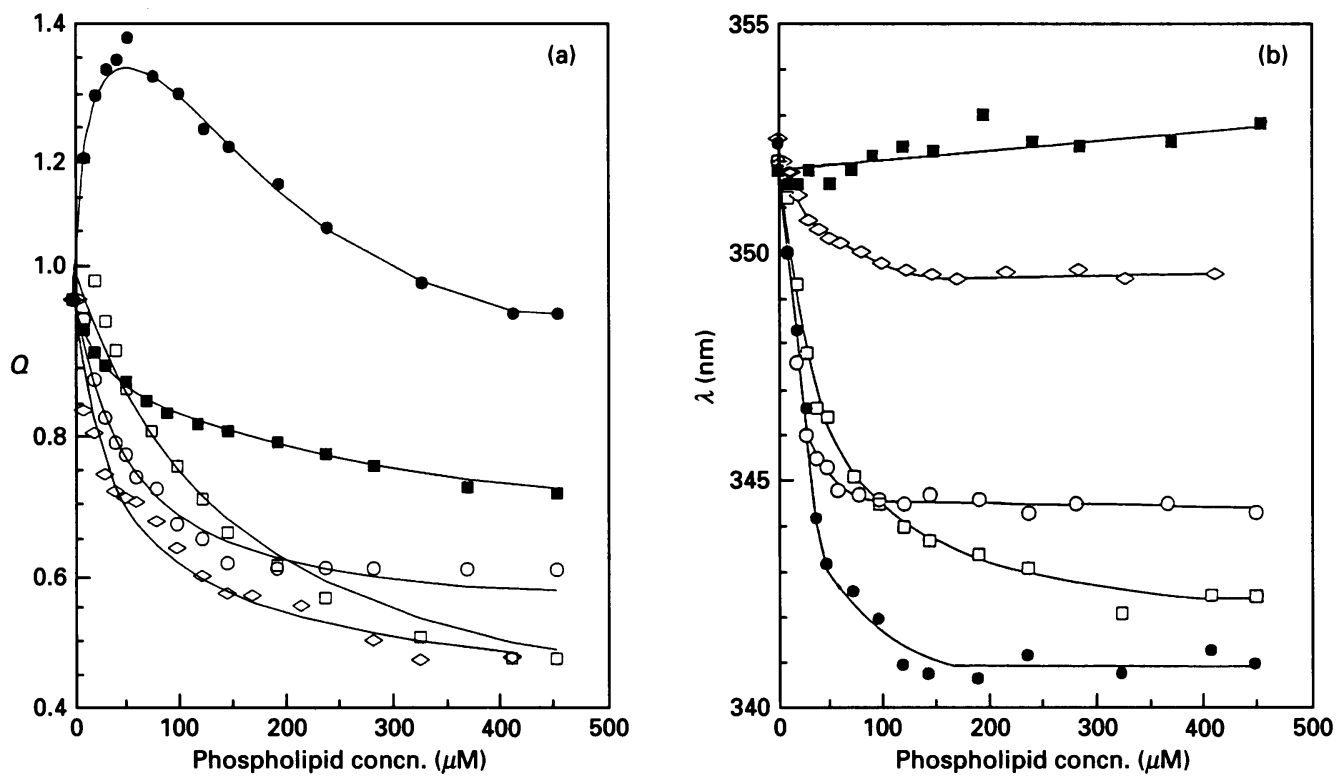

Figure 1 Spectrofluorimetric titration of caldesmon with vesicles composed of various phospholipids

(a) Relative quantum yield $(Q)$ and (b) spectrum position $(\lambda)$ of the tryptophan fluorescence of caldesmon titrated with PS $(O), P C(\square), C L(\diamond), P I(\square)$ and PA $(O)$ vesicles. Excitation was at $297 \mathrm{~nm}$. Reaction conditions were as follows: $2 \mu \mathrm{M}$ caldesmon in $20 \mathrm{mM}$ Tris/HCl (pH 7.5), $0.5 \mathrm{mM} \mathrm{EGTA,} 1 \mathrm{mM} \mathrm{NaN}{ }_{3}$ and $1 \mathrm{mM}$ 2-mercaptoethanol. The S.D. value for $Q$ is not higher than $5 \%$, whereas for $\lambda$ S.D. $= \pm 1 \mathrm{~nm}$.

mon with a given concentration of lipid to the quantum yield of fluorescence of caldesmon alone. The position of the middle of the chord drawn at the $80 \%$ level of the maximal intensity was taken as a measure of the spectral position $(\lambda)$. All spectra were measured in solutions containing buffer $\mathrm{A}$.

The experimental results of the fluorescence quantum yield were fitted by a theoretical curve computed according to the simplest one-site binding scheme shown in eqn. (1):

$$
\mathrm{CaD}+\mathrm{Lp} \stackrel{K_{\text {ass. }}}{\rightleftharpoons} \mathrm{CaD} \cdot \mathrm{Lp}
$$

where $\mathrm{CaD}$ is caldesmon, $\mathrm{Lp}$ is lipid, $K_{\mathrm{ass}}$ the association constant, and $\mathrm{CaD} \cdot \mathrm{Lp}$ is caldesmon-lipid complex. If the fitting of the experimental results with a theoretical curve was not satisfactory, in particular where the curves showed biphasic character, we used a scheme implicating two-types of binding sites:

$$
\begin{aligned}
& \mathrm{CaD}+\mathrm{Lp} \stackrel{K_{\text {ass. } 1}}{\rightleftharpoons} \mathrm{CaD} \cdot \mathrm{Lp} \\
& \mathrm{CaD} \cdot \mathrm{Lp}+n \cdot \mathrm{Lp} \stackrel{K_{\text {ass. } 2}}{\rightleftharpoons} \mathrm{CaD} \cdot(\mathrm{Lp})_{n+1}
\end{aligned}
$$

where $n$ stands for the number of binding domain(s) on caldesmon with $K_{\text {ass.2 }}$ for the lipid vesicle(s). The fitting of the experimental results to theoretical curves was carried out with a computer using a non-linear regression scheme [24].

\section{Affinity chromatography}

Affinity chromatography was performed on a calmodulinSepharose 4B column. Calmodulin was coupled to $\mathrm{CNBr}$ activated Sepharose 4B (Pharmacia) according to the procedure outlined by Pharmacia. Caldesmon was bound to the affinity column $(1 \mathrm{~cm} \times 8 \mathrm{~cm})$ in buffer containing $20 \mathrm{mM}$ Tris $/ \mathrm{HCl}$ (pH 7.5), $50 \mathrm{mM} \mathrm{NaCl}, 3 \mathrm{mM} \mathrm{MgCl}_{2}, 1 \mathrm{mM} \mathrm{NaN}, 1 \mathrm{mM} 2-$ mercaptoethanol (buffer B) and $2 \mathrm{mM} \mathrm{CaCl}$. After washing with buffer B supplemented with $150 \mathrm{mM} \mathrm{NaCl}$, and $0.2 \mathrm{mM}$ $\mathrm{CaCl}_{2}$, the column was equilibrated with buffer $\mathrm{B}$ containing $0.2 \mathrm{mM} \mathrm{CaCl}_{2}$ alone. The phospholipid vesicle samples $(5 \mathrm{ml})$, in the same buffer, were applied to the column, which was subsequently eluted with buffer B containing $0.2 \mathrm{mM} \mathrm{CaCl}_{2}$ and buffer B containing $1 \mathrm{mM}$ EGTA.

\section{RESULTS}

\section{Interaction of caldesmon with vesicles composed of various phospholipids}

It is known that smooth muscle caldesmon contains five tryptophan residues per molecule [19,25], and all of them are situated at the surface of the protein [16]. Therefore, fluorescence spectroscopy is a well-suited technique for studying interactions of caldesmon with phospholipid vesicles.

Figure 1 shows the results of the spectrofluorimetric titration of caldesmon with different phospholipids. In most cases the interaction of caldesmon with phospholipids is concomitant with a decrease in the relative quantum yield $(Q)$ of tryptophan fluorescence, except for with PS, where there was an increase in $Q$ value at low concentrations (Figure la). At higher concentrations of PS (above $50 \mu \mathrm{M}$ ) the decrease in $Q$ is similar to that observed for CL, PI and PA and significantly higher than that for PC. Quantitative analysis of the results revealed that the binding of anionic phospholipids (net charge) and zwitterionic PC to caldesmon is relatively weak $\left(K_{\text {ass. }} \approx 10^{4} \mathrm{M}^{-1}\right)$ (Table 1$)$. However, in the case of PS, the binding affinity is an order of magnitude higher $\left(K_{\text {ass. }}=1.45 \times 10^{5} \mathrm{M}^{-1}\right)$ than those for the other phospholipids. Strong binding of PS is saturated at $50 \mathrm{~mol}$ of this phospholipid per mol of caldesmon. The second type of weak binding sites $\left(K_{\text {ass. }}=4.6 \times 10^{3} \mathrm{M}^{-1}\right)$ requires much more PS for saturation.

Interaction of caldesmon with all anionic phospholipids examined is accompanied by a short-wavelength shift of the tryptophan 
Table 1 Binding parameters of caldesmon to vesicles composed of various phospholipids or their mixtures

\begin{tabular}{llll}
\hline Vesicle composition & $10^{-5} \times K_{\text {ass } 1}\left(\mathrm{M}^{-1}\right)$ & $10^{-3} \times K_{\text {ass } 2}\left(\mathrm{M}^{-1}\right)$ & $n$ \\
\hline PS & $1.45 \pm 0.12$ & $4.6 \pm 0.4$ & 2 \\
PC & $0.31 \pm 0.03$ & $3.4 \pm 0.3$ & 2 \\
$C L$ & $0.22 \pm 0.02$ & - & - \\
PA & $0.20 \pm 0.02$ & - & - \\
PI & $0.10 \pm 0.01$ & - & - \\
$P S+P C(1: 1, \mathrm{~mol} / \mathrm{mol})$ & $1.45 \pm 0.15$ & $5.1 \pm 0.4$ & - \\
$P S+P C(1: 4, \mathrm{~mol} / \mathrm{mol})$ & $0.20 \pm 0.02$ & - & - \\
$D C P+P C(1: 4, \mathrm{~mol} / \mathrm{mol})$ & $0.10 \pm 0.02$ & - & \\
& & & \\
\hline
\end{tabular}

fluorescence spectrum (Figure 1b), indicating that the environment of the tryptophan residues becomes more hydrophobic. The effectiveness of these phospholipids in changing the spectrum decreases in the following order: PS $>$ PI $>$ PA $>$ CL. In the case of $\mathrm{PC}$, the spectrum of caldesmon does not alter appreciably.

\section{Interaction of caldesmon with vesicles composed of a mixture of phospholipids}

To study the effect of the surface charge of phospholipid vesicles on their interaction with caldesmon, the composition of vesicles was modified. Figure 2 shows the interaction of caldesmon with vesicles composed of PC and PS or PC and dicetylphosphate (DCP). The $Q$ value of the mixture containing caldesmon and vesicles composed of equimolar amounts of PC and PS is identical to that obtained for caldesmon and PS alone, i.e. it increases at low concentrations and decreases at high concentrations of phospholipid (see Figure 1a). The calculated $K_{\text {ass. }}$ value of caldesmon binding to vesicles containing a $1: 1$ mixture of PC and PS is identical to that of caldesmon to PS vesicles (Table 1). At a 4:1 molar mixture of PC and PS, the $Q$ value gradually increases in the whole range of phospholipid mixtures used. The curve seems to correspond to the ascending part of PS and PC + PS (1:1 molar mixture) curves but, due to the much lower content of PS in vesicles, it is significantly less steep. The $Q$ value of caldesmon with a 4:1 molar mixture of PC and DCP is lower than that for a mixture composed of PC and PS at the same ratio. In the latter two cases the increase in the negative charge on the surface of PC vesicles, caused by incorporation of either anionic PS or DCP, does not appreciably affect their $K_{\text {ass. }}$ to caldesmon (Table 1).

A shift in the maximum value of the tryptophan fluorescence of caldesmon towards shorter wavelengths which is demonstrated for 4:1 molar mixtures of PC and PS or PC and DCP is the same, although it is significantly lower than that registered at a $1: 1$ molar ratio of PC to PS (Figure 2b).

\section{Influence of $\mathrm{NaCl}$ on the interaction of caldesmon with PS vesicles}

The effect of ionic strength on the binding of PS vesicles to caldesmon is shown in Figure 3. Up to physiological ionic strength the effect of $\mathrm{NaCl}$ is rather small; however, over $150 \mathrm{mM}$ $\mathrm{NaCl}$ there is a dramatic decrease in the affinity of PS for caldesmon (Figure 3a). At physiological ionic strength the value of $K_{\text {ass. }}$ for the binding of PS to caldesmon is $1.0 \times 10^{5} \mathrm{M}^{-1}$, i.e. about one third lower than that determined in the absence of $\mathrm{NaCl}$, while at $250 \mathrm{mM} \mathrm{NaCl} K_{\text {ass. }}$ is an order of magnitude lower.

Calculation of the surface potential of PS vesicles $\left(\psi_{0}\right)[26]$ at various $\mathrm{NaCl}$ concentrations showed that its most dramatic decrease occurs between 0 and $10 \mathrm{mM} \mathrm{NaCl}$; above this concentration of $\mathrm{NaCl}$ there is still a gradual decrease of the potential, but at a much slower rate. Because of a marked difference between the dependence of $K_{\text {ass. }}$ and $\psi_{0}$ versus $\mathrm{NaCl}$ concentration we can conclude that the effect of $\mathrm{NaCl}$ on the interaction between caldesmon and the phospholipid may be only partially due to the screening of the surface potential of PS
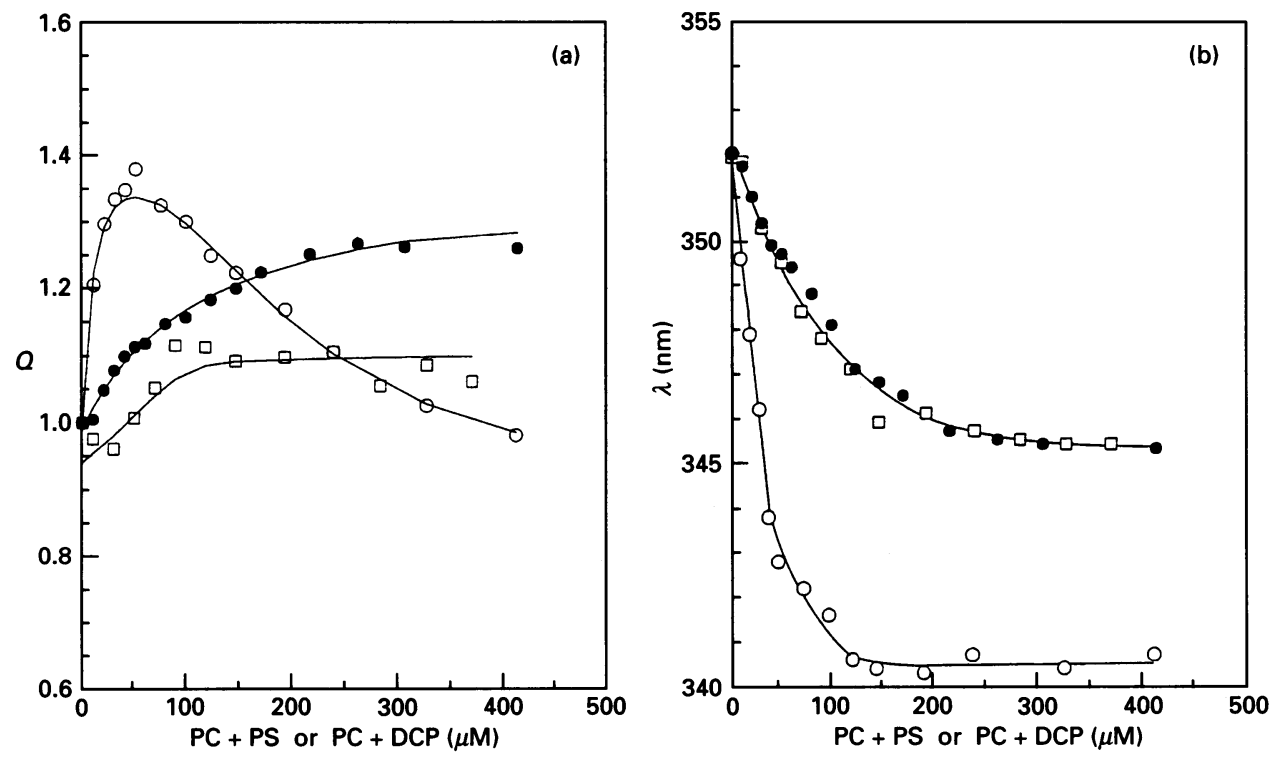

Figure 2 Spectrofluorimetric titration of caldesmon with vesicles composed of mixed phospholipids

(a) Relative quantum yield $(Q)$ and (b) spectrum position $(\lambda)$ of the tryptophan fluorescence of caldesmon titrated with a mixture (mol/mol) of PC and PS (1:1) (O), PC and PS (4:1) (O) or PC and DCP $(4: 1)(\square)$ vesicles. Conditions and S.D. values as given in the legend to Figure 1. 


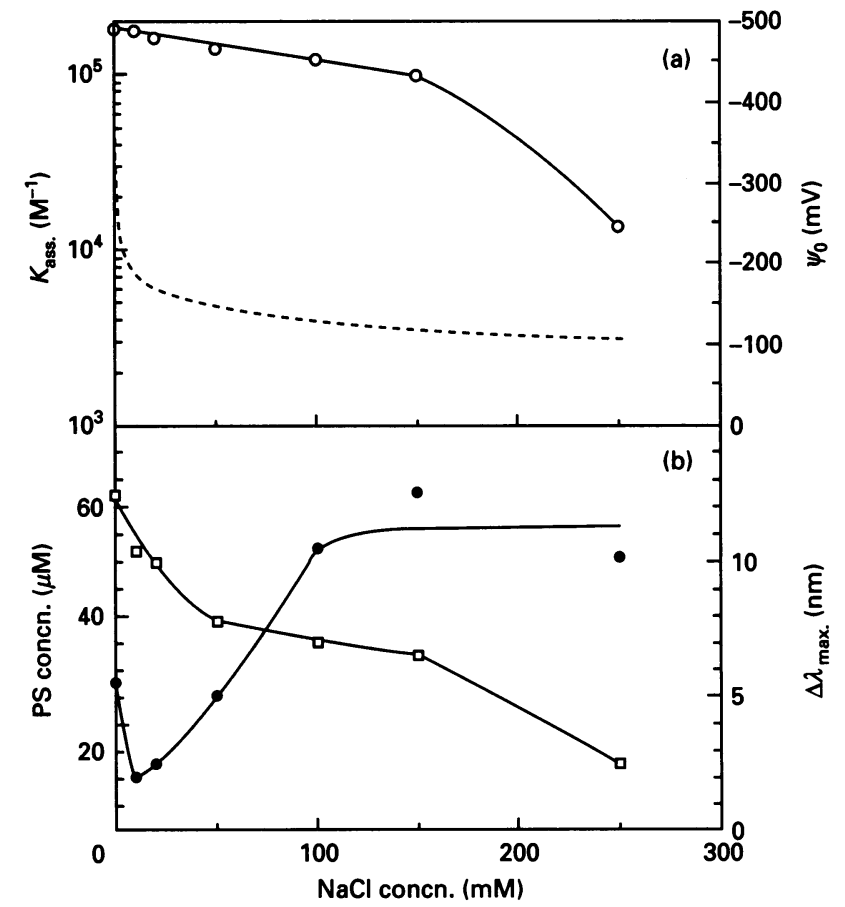

Figure 3 Spectrofluorimetric titration of caldesmon-PS vesicle complex with $\mathrm{NaCl}$

(a) Dependence on $\mathrm{NaCl}$ concentration of $K_{\text {ass. }}$ values of caldesmon-PS vesicles complex (O), S.D. values of about $5 \%$, and surface potential values of PS were calculated according to [26] $(--)$ as well as (b) the value of the short-wavelength shift $\left[\Delta \lambda=(\lambda)_{\mathrm{NaCl}}-(\lambda)_{\mathrm{NaCl} P S}\right]$ of the tryptophan fluorescence spectrum of caldesmon ( $\square$ ), S.D. $= \pm 1 \mathrm{~nm}$ and the concentration of PS required for half this short-wavelength shift $(O)$, S.D. $= \pm 5 \mu \mathrm{M}$. Conditions were as described in the legend to Figure 1.

vesicles. This may be true especially for high $\mathrm{NaCl}$ concentrations where a sharp decrease of $K_{\text {ass. }}$ occurs.

Parallel determination of the effect of $\mathrm{NaCl}$ on the value of the short-wavelength shift of the tryptophan fluorescence spectrum of caldesmon $(\Delta \lambda)$ and on the amount of PS required to obtain half the maximum value, allowed us to evaluate the contribution of hydrophobic interactions in the overall caldesmon-PS interactions. Figure 3(b) indicates that PS-induced short-wavelength shift of the tryptophan spectrum of caldesmon is maximal in the absence of $\mathrm{NaCl}$ and gradually decreases with an increase of $\mathrm{NaCl}$ concentration. At $50 \mathrm{mM} \mathrm{NaCl}$ it reaches half of its maximum value and this level is maintained up to $150 \mathrm{mM} \mathrm{NaCl}$. Further enhancement of the $\mathrm{NaCl}$ concentration causes a marked decrease in the shift of the caldesmon spectrum, probably due to partial dissociation of the hydrophobic interactions between PS and caldesmon. However, it is worthwhile noting that some hydrophobic interactions are preserved even at $250 \mathrm{mM} \mathrm{NaCl}$.

As shown from the curve of dependence of the amount of PS required to produce half the maximum shift of the caldesmon tryptophan fluorescence spectrum on $\mathrm{NaCl}$ concentration, the lipid is most effective between $10 \mathrm{mM}$ and $20 \mathrm{mM} \mathrm{NaCl}$. With the increase in $\mathrm{NaCl}$ concentration up to $100 \mathrm{mM}$ its effectiveness is decreased and does not change above this concentration of $\mathrm{NaCl}$.

\section{Competition of PS and calmodulin for the binding of caldesmon}

A $5 \mathrm{mM}$ solution of PS vesicles eluted all caldesmon conjugated with a calmodulin-affinity column in the presence of $\mathrm{Ca}^{2+}$,

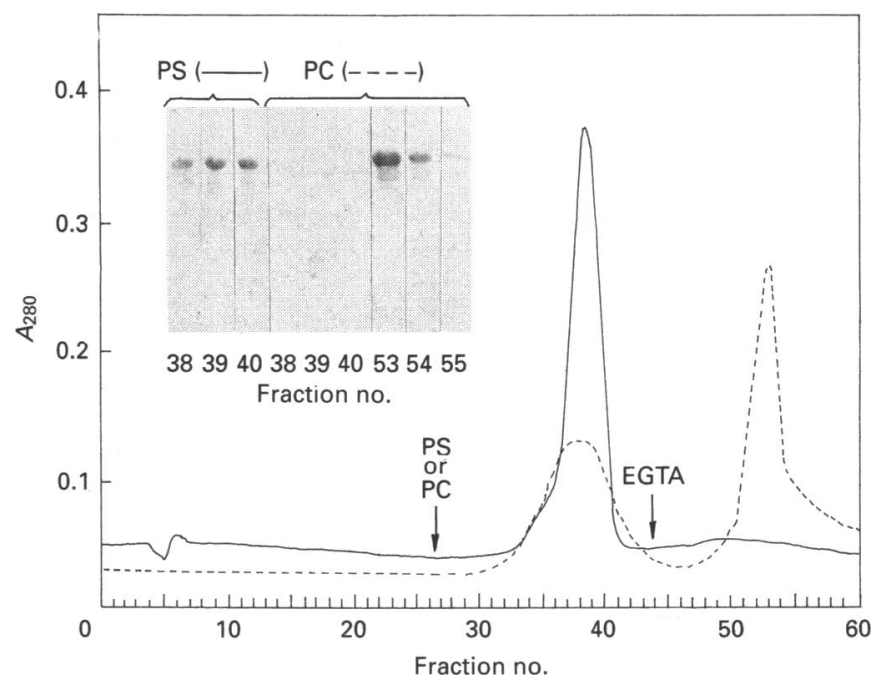

Figure 4 Interaction of PS vesicles with caldesmon bound to calmodulinaffinity column

Caldesmon in buffer containing $20 \mathrm{mM}$ Tris/HCl (pH 7.5), $50 \mathrm{mM} \mathrm{NaCl}, 3 \mathrm{mM} \mathrm{MgCl}, 1 \mathrm{mM}$ $\mathrm{NaN}_{3}, 1 \mathrm{mM}$ 2-mercaptoethanol (buffer $\mathrm{B}$ ), and $2 \mathrm{mM} \mathrm{CaCl}{ }_{2}$, was applied on a calmodulin-affinity column $(1 \mathrm{~cm} \times 8 \mathrm{~cm})$. The column was then washed with buffer $B$ supplemented with $150 \mathrm{mM}$ $\mathrm{NaCl}$ and $0.2 \mathrm{mM} \mathrm{CaCl}_{2}$, and equilibrated with buffer $B$ containing $0.2 \mathrm{mM} \mathrm{CaCl}_{2} . A$ sample of PS or PC (5 mM) in the same buffer was applied to the column, which was washed

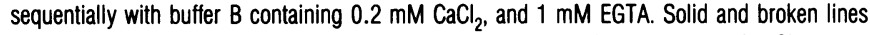
show the elution profiles after applying PS or PC to the column respectively. Since u.v. absorbance was not corrected for light scattering from lipid solution, selected fractions $(2 \mathrm{ml})$ were subjected to SDS/PAGE (inset).

whereas PC used at the same concentration as PS was unable to extract caldesmon. In the latter case all the protein was eluted with buffer containing $1 \mathrm{mM}$ EGTA (Figure 4).

\section{DISCUSSION}

Sequence analysis of caldesmon showed that $50 \%$ of its amino acid residues are charged $[19,25]$. Therefore, the electrostatic interaction of this protein with charged phospholipids is expected. Depending on the charge distribution on the surface of lipid vesicles, three types of interaction with caldesmon can be considered: first, that involving pure anionic phospholipids (PI, PA, and CL); secondly, that to PS, that besides two negative charges contains one positive charge; and thirdly, that to zwitterionic PC, which despite its zero net charge can also interact electrostatically [27].

The results presented here demonstrate that among vesicles composed of various individual phospholipids the strongest complex with caldesmon is formed by PS vesicles. It indicates that the distribution of the charged groups on the PS molecule is the most suitable for producing an electrostatic interaction with caldesmon. The importance of proper distribution of the charge on the surface of the phospholipid vesicles is confirmed by the fact that the incorporation of $20 \mathrm{~mol} \%$ of the negatively charged PS or DCP into PC containing vesicles does not increase their binding affinity to caldesmon, and that the binding affinity of the vesicles composed of a 1:1 molar mixture of PC and PS is the same as that obtained for vesicles composed of PS alone (Table 1). The existence of electrostatic interaction between caldesmon and PS vesicles is shown by the observed decrease in affinity of binding at high ionic strength. Electrostatic coupling, as judged from short-wavelength shift of the tryptophan emission spectrum of caldesmon is accompanied by hydrophobic interaction between the caldesmon domain(s) containing tryptophan residues 
and the hydrophobic part of the lipid bilayer (acyl chains of the phospholipid).

The different changes in the apparent quantum yield of tryptophan fluorescence $(Q)$ upon binding of various lipids with caldesmon may suggest that different tryptophan residues of this protein are involved in this binding. It was shown previously [16] that among five tryptophan residues of caldesmon four are accessible to free water and one is in contact with bound water. According to Burstein et al. [28], who measured fluorescence parameters of the tryptophan spectra for a number of proteins (taking into account the environment of tryptophan residues), transference of the tryptophan residues from the unstructured to structured environment is accompanied by an increase of $Q$ value and short-wavelength shift of the spectrum. Further transference of tryptophan residues to the hydrophobic environment is concomitant with a decrease in $Q$ value and intensification of the blue shift of the spectrum. These two-step changes in tryptophan environment seem to take place upon binding of caldesmon to vesicles composed of PS and a 1:1 molar mixture of PS and PC. The only one-step changes probably occur in the case of the other phospholipids (PA, PI, PC and CL).

According to Vorotnikov and Gusev [2] the site(s) of interaction with lipids is located in the chymotryptic $40 \mathrm{kDa}(34 \mathrm{kDa}$ from the sequence) C-terminal fragment of caldesmon. Our preliminary results showed that the $25 \mathrm{kDa}$ C-terminal fragment does not bind PS. Thus, the binding site has to be located at the $\mathrm{N}$-terminal region of the $34 \mathrm{kDa} \mathrm{C}$-terminal fragment. The most favourable site for PS binding would be the sequence between Asp-510 and Lys-565 that has an extremely dense charge distribution [19]. Moreover, this part of the caldesmon molecule could form an amphipathic helix, with a surface containing only oppositely charged and hydrophobic residues; such a helix has been postulated to interact superficially with membranes [29].

The second weak binding site registered for PS and PC vesicles could be located in the $\mathrm{N}$-terminal region of the caldesmon molecule. Accumulation in this region of positively charged amino acid residues, and the presence of a tryptophan residue, the fluorescence of which can record the binding, might be compatible with this concept.

PS causes competitive displacement of calmodulin from its complex with caldesmon (Figure 4). In the sequence of the $34 \mathrm{kDa}$ C-terminal fragment of caldesmon the predicted PSbinding site is not close to the calmodulin-binding site [19]. However, according to a recent proposal of Wang et al. [30] the calmodulin-binding site is constructed from two relatively distant C-terminal sequences of caldesmon (amino acid residues 446-459 and 629-666). It is suggested that in the tertiary structure of the protein these sequences are close to each other. Therefore, binding of PS at the site located between them, due to steric hindrance or conformational changes in the polypeptide chain, can destroy the calmodulin-binding site. The proximity of PSand calmodulin-binding sites on caldesmon is confirmed by the fact that, upon interaction with caldesmon, both PS and calmodulin cause transference of its $\mathrm{C}$-terminal tryptophan residues (Trp-674, Trp-707 and Trp-737) into a more hydrophobic environment [16,31] (Figure 1a).

Recently it was shown that phosphorylation of caldesmon by $\mathrm{Ca}^{2+}$ - and phospholipid-dependent protein kinase $\mathrm{C}$ significantly decreases its affinity for phospholipid [32]. The location of the two phosphorylated serine residues (Ser-587 and Ser-726) in the C-terminal region of caldesmon $[33,34]$ is consistent with the region of caldesmon predicted to interact with PS.

According to Rodaway et al. [35] the family of a diverse population of membrane-associated cytoplasmic proteins, in- cluding Acanthamoeba myosin I [36], share a sequence of 50 amino acid residues. This sequence is, however, absent from the predicted PS-binding sequence of caldesmon.

In contrast with PC, which is the predominating constituent of the outer lipid bilayer, PS is located mainly in the inner half of the lipid bilayer [37]. Thus, it is possible that this phospholipid provides the site of anchorage of caldesmon, and indirectly actin filaments, to the plasma membrane. This attachment could be controlled through phosphorylation of caldesmon by protein kinase C. In support of this view is evidence for protein kinase $\mathrm{C}$ phosphorylation of caldesmon in vivo $[38,39]$ as well as the presence of caldesmon at the periphery of adrenal chromaffin cells during the secretory process [40], in splenic T-lymphocytes during surface-receptor capping [7], and also in the ruffing membranes of fibroblasts [4].

We gratefully acknowledge the help of Ms. A. Dygas in purification of PS. We also thank Professor L. Wojtczak for helpful comments regarding Gouy-Chapman theory.

\section{REFERENCES}

1 Isenberg, G. (1991) J. Muscle Res. Cell Motil. 12, 136-144

2 Vorotnikov, A. V. and Gusev, N. B. (1990) FEBS Lett. 277, 134-136

3 Sobue, K., Muramoto, Y., Fujita, M. and Kakiuchi, S. (1981) Proc. Natl. Acad. Sci. U.S.A. 78, 5652-5655

4 Bretscher, A. and Lynch, W. (1985) J. Cell. Biol. 100, 1656-1663

5 Dingus, J., Hwo, S. and Bryan, J. (1986) J. Cell Biol. 102, 1748-1757

6 Sobue, K., Tanaka, T., Kana, K, Ashino, N. and Kakiuchi, S. (1985) Proc. Natl. Acad. Sci. U.S.A. 82, 5025-5029

7 Mizushima, Y., Kanda, K., Hamaoka, T., Fujiwara, H. and Sobue, K. (1987) Biomed. Res. 8, 73-78

8 Pritchard, K. and Moody, C. J. (1986) Cell Calcium 7, 309-327

9 Chalovich, J. M. (1988) Cell Biophys. 12, 73-85

10 Gałązkiewicz, B., Mossakowska, M., Osińska, H. and Dąbrowska, R. (1985) FEBS Lett. 184, 144-149

11 Gałązkiewicz, B., Belagyi, J. and Dąbrowska, R. (1989) Eur. J. Biochem. 181, $607-614$

12 Gałazkiewicz, B., Buss, F., Jockusch, B. M. and Dabrowska, R. (1991) Eur. J. Biochem. 195, 607-614

13 Hartwig, J. H., Chambers, L. A., Hopica, K. L. and Kwiatkowski, D. J. (1989) J. Cell. Biol. 109, 1571-1579

14 Lassing, I. and Lindberg, V. (1988) Exp. Cell Res. 174, 1-15

15 Bretscher, A. (1984) J. Biol. Chem. 259, 12873-12880

16 Czuryło, E. A., Emelyanenko, V. I., Permyakov, E. A. and Dąbrowska, R. (1991) Biophys. Chem. 40, 181-188

17 Gopalakrishna, R. and Anderson, W. B. (1982) Biochem. Biophys. Res. Commun. 104, 830-836

18 Laemmli, U. K. (1970) Nature (London) 227, 680-685

19 Bryan, J., Imai, M., Lee, R., Moore, P., Cook, R. G. and Lin, W. G. (1989) J. Biol. Chem. 264, 13873-13879

20 Folch, J. (1942) J. Biol. Chem. 146, 35-44

21 Comfurius, P. and Zwaal, R. F. A. (1977) Biochim. Biophys. Acta 488, 36-42

22 Singleton, W. S., Gray, M. S., Brown, M. L. and White, J. L. (1965) J. Am. Oil Chem. Soc. 42, 53-56

23 Rouser, G., Fleischer, S. and Yamamoto, A. (1970) Lipids 5, 494-496

24 Reich, J. G., Wangerman, G., Falk, M. and Rohde, K. (1972) Eur. J. Biochem. 26, 368-379

25 Hayashi, K., Kanda, K., Kimizuka, F., Kato, I. and Sobue, K. (1989) Biochem. Biophys. Res. Commun. 164, 503-511

26 Wojtczak, L. and Nałecz, M. J. (1985) in Structure and Properties of Cell Membranes (Benga, G., ed.), vol. 2, pp. 215-242, CRC Press, Boca Raton, Florida

27 Tocanne, J.-F. and Teissié, J. (1990) Biochim. Biophys. Acta 1031, 111-142

28 Burstein, E. A., Vedenkina, N. S. and Ivkova, M. N. (1973) Photochem. Photobiol. 18, 263-279

29 Morrisett, J. D., Jackson, R. L. and Gotto, A. M., Jr. (1975) Annu. Rev. Biochem. 44, 183-207

30 Wang, C.-L. A., Wang, L.-W. C., XU, S., Lu, R. C., Saavedra-Alanis, V. and Bryan, J. (1991) J. Biol. Chem. 266, 9166-9172

31 Shirinsky, V. P., Bushueva, T. L. and Frolova, S. I. (1988) Biochem J. 255, 203-208

32 Vorotnikov, V. A., Bogatcheva, N. V. and Gusev, N. B. (1992) Biochem. J. 284, 911-916

33 Adam, L. P. and Hathaway, D. R. (1990) Biophys. J. 57, 150

34 Ikebe, M. and Hornick, T. (1991) Arch. Biochem. Biophys. 288, 538-542 
35 Rodaway, A. F., Sternberg, M. J. and Bentley, D. L. (1989) Nature (London) 342, 624

36 Adams, R. and Pollard, T. (1989) Nature (London) 340, 565-568

37 Op den Kamp, J. A. F. (1979) Annu. Rev. Biochem. 48, 47-71

38 Litchfield, D. W. and Ball, E. H. (1987) J. Biol. Chem. 262, 8056-8060
39 Adam, L. P., Milio, L., Brengle, B. and Hathaway, D. R. (1990) J. Mol. Cell Cardiol. 22, 1017-1027

40 Burgoyne, R. D., Check, T. R. and Horman, K.-M. (1986) Nature (London) 319, 68-73

Received 23 April 1992/15 October 1992; accepted 26 October 1992 